\title{
UNQUENCHED QUARK MODEL DESCRIPTION OF CHARMONIUM
}

\author{
P. GONZÁLEZ \\ Departamento de Física Teórica, Universidad de Valencia (UV) and IFIC (UV-CSIC), \\ Valencia, Spain. \\ pedro.gonzalez@uv.es
}

\begin{abstract}
We show that the charmonium spectrum may be reproduced to a good precision by means of a semirelativistic quark model approach based on a universal static potential effectively incorporating effects from sea quark-antiquark pairs. Detailed predictions for upcoming data are derived.
\end{abstract}

PACS numbers: 14.40.-n,12.39.-x

In the last ten years the interest in the charmonium spectrum has been renewed as a consequence of the experimental observation of many new resonances. So fifteen new charmonium states have been cataloged during this time in the Particle Data Book Review ${ }^{1}$. Most of these states have masses above the threshold for OZI allowed decay to open heavy flavor mesons. Concerning the quantum numbers of these states above threshold they are only known for those of them extracted from Initial State Radiation (ISR) processes which can be unambiguosly identified as $J^{P C}=1^{--}$ resonances. Therefore a basic requirement to any reliable spectroscopic model is the correct description of these $1^{--}$states as well as of the well established resonances below threshold.

Unfortunately lattice-QCD calculations are not still refined to provide a precise description of states above threshold. Instead we develop here an unquenched quark model approach. In order to take into account the coupling to meson-meson components which becomes relevant above threshold we build up an unquenched quarkantiquark potential effectively incorporating sea quark effects. Thus the coupling to meson-meson components comes naturally from the recombination of the valence quark and antiquark with sea antiquark-quark pairs. This idea is not new. In the nineties Isgur and collaborators pointed out that in the adiabatic approximation most of the effects of $q \bar{q}$ loops on spectroscopy, reflecting effects of meson-meson couplings, could be absorbed into a (renormalized) static interquark potential ${ }^{2}$. However, the long distance behavior of the renormalized static potential and its connection with the static interaction one may derive from QCD when quark loops are included was not investigated. We investigate this connection and proceed the other way around. We require the quark-antiquark potential to have the same long 
distance behavior as the OGE static interaction from QCD (including quark loops) and assume that such unquenched potential may represent the average effect of meson-meson couplings. Then we use this potential in a semirelativistic approach to the static spectrum and particularize it to charmonium.

The investigation, through the solutions of the Schwinger-Dyson Equations (SDE), of the non perturbative running of $\alpha_{s}\left(\alpha_{s}\right.$ is proportional to the square of the quark-gluon-quark coupling) shows a freezing of the coupling in the infrared that can be parametrized as ${ }^{3}$

$$
\alpha_{s}\left(Q^{2}\right)=\frac{4 \pi}{11 \ln \left(\frac{Q^{2}+\xi M_{g}^{2}}{\Lambda^{2}}\right)-\frac{2}{3} n_{f} \ln \left(\frac{Q^{2}+\xi m_{q}^{2}}{\Lambda^{2}}\right)}
$$

where $n_{f}$ stands for the number of flavours (in the quark loops), $M_{g}\left(Q^{2}\right)$ for a dynamic gluon mass ( $\mathrm{see}^{4}$ for an approximate expression of it and for comparison with other definitions of the gluon mass), $m_{q}^{2}\left(Q^{2}\right)$ for the dynamic quark mass (see ${ }^{5}$ for its expression from instanton model and lattice calculations), $\Lambda$ for the QCD scale parameter and $\xi$ for a constant to be fitted from data.

From $\alpha_{s}$ the OGE diagram for quark-antiquark scattering can be evaluated. By equating the scattering amplitude (for no energy transfer between quark-antiquark and neglecting spin dependent terms) with the static potential between quark and antiquark inside a meson one obtains $V_{s t}\left(\vec{q}^{2}\right)=-\frac{C_{s t} \alpha_{s}\left(\vec{q}^{2}\right)}{\vec{q}^{2}}$ where $\vec{q}^{2}=-\vec{Q}^{2}$ and $C_{s t}$ is a constant.

If one considers first the quenched approximation (no quark loops) $n_{f}=0$ and takes as an average in the whole $Q^{2}$ interval $\xi M_{g}^{2}\left(Q^{2}\right) \sim \Lambda^{2}$ one obtains a Richardson potential of the form $V_{R}\left(\vec{q}^{2}\right)=-\frac{C_{R}}{\vec{q}^{2} \ln \left(1+\vec{q}^{2} / \Lambda^{2}\right)}$ with $C_{R}$ a constant. For $\vec{q}^{2} \gg \Lambda^{2}$ the corresponding short distance behavior is approximately coulombic with a logarithmic dependence of the coupling strength to account for asymptotic freedom. For $\vec{q}^{2} \ll \Lambda^{2}$ one has a linear confining potential (linearly increasing with the quark-antiquark distance). A simplified interpolating form between these limits in configuration space corresponds to the so called Cornell potential reading (up to an additive constant) $V_{C}(r)=\sigma r-\frac{k}{r}$. This potential form provides a good fit to the well known experimental spin averaged heavy quarkonia levels up to the threshold for OZI allowed decay to open heavy flavor mesons ${ }^{6}$.

In order to incorporate quark loops effects let us take $n_{f} \neq 0$. By taking as averages in the whole $Q^{2}$ interval $\xi M_{g}^{2}\left(Q^{2}\right) \sim \Lambda^{2}$ and $\xi m_{q}^{2}\left(Q^{2}\right) \sim a \Lambda^{2}$ being $a$ a constant presumably lower than 1 (one may expect the quark mass to be lower than the gluon mass $\left.^{3}\right)$ the resulting static potential is $V_{s t} \sim-\frac{C}{\vec{q}^{2}\left[11 \ln \left(1+\frac{\vec{q}^{2}}{\Lambda^{2}}\right)-\frac{2 n_{f}}{3} \ln \left(a+\frac{\vec{q}^{2}}{\Lambda^{2}}\right)\right]}$. In the limit $\vec{q}^{2} \gg \Lambda^{2}$ one has again a coulombic potential with a strength running as the perturbative expression of $\alpha_{s}\left(\left(\alpha_{s}\right)_{\text {per }}=4 \pi / \beta_{0} \ln \left(\vec{q}^{2} / \Lambda^{2}\right)\right.$ with $\left.\beta_{0}=11-(2 / 3) n_{f}\right)$. Quite surprisingly for $\vec{q}^{2} \ll \Lambda^{2}$ one also obtains (if $a<1$ ) a coulombic potential $V_{s t} \rightarrow \frac{C_{s}}{\left(\frac{2 n_{f}}{3} \ln a\right) \vec{q}^{2}}$ indicating that as a result of sea pair effects the linear confinement may become a Coulomb interaction. This shocking long-distance behavior seems to have some phenomenological support. Some years ago it was realized that 
the high excited (large-sized) light-quark meson spectrum suggests a hydrogenlike degeneracy ${ }^{7}$.

An interpolating form between these two limiting behaviors has been recently proposed $^{8}$

$$
V(r)=\left(\sigma r-\frac{k}{r}\right)\left(1-e^{-\frac{\nu}{r}}\right) \equiv\left(\sigma r-\frac{k}{r}\right) F(r)
$$

where $k$ is a a $\vec{q}^{2}$ dependent parameter that will be fixed as a constant in each static meson sector from data and $F(r) \equiv\left(1-e^{-\frac{\nu}{r}}\right)$ is the unquenching factor. Let us note that $F(r)$ is very close to 1 for $0.2 \mathrm{fm}<r<1 \mathrm{fm}$ ( $\nu$ is fixed once for all from the fit to the static light-quark meson spectrum to $\nu=2.3 \mathrm{fm}$ ) so that the potential corresponds to the Cornell-like form. On the other hand for $r \gg \nu$ one has $F(r) \rightarrow \frac{\nu}{r}-\frac{\nu^{2}}{2 r^{2}}$ from which an asymptotic $(r \rightarrow \infty)$ coulombic behavior is derived $V(r) \rightarrow \sigma \nu-\frac{\sigma \nu^{2}}{2 r}$. Notice that this long-distance coulombic behavior comes from the effective unquenching of the linear confinement as the dependence on $\sigma$ makes manifest and has nothing to do with the perturbative OGE coulombic interaction, with strength $k$, dominating at short distances and giving rise at long distances to a subdominant $1 / r^{2}$ behavior). For a comparison of $F(r)$ to other unquenching proposals see ${ }^{8}$.

It should be remarked that $V(r)$ is finite at infinite distance: $V(\infty)=\sigma \nu$. Therefore no additive constant will be needed to get the spectral masses which will have an upper energy bound $\left(M_{\text {Limit }}\right)_{q \bar{q}}=m_{q}+m_{\bar{q}}+\sigma \nu$. This limiting mass represents the upper limit of the domain of applicability of our unquenched model. Beyond this mass the effect of meson-meson couplings can not be effectively absorbed in the valence quark-antiquark static potential since the probability of having this valence quark-antiquark component vanishes. String breaking takes place and the description has to be done in terms of $2 q 2 \bar{q}$ or meson-meson components.

In order to apply this potential to the description of charmonium we should realize that a nonrelativistic treatment is expected to be quite crude since quark velocities can be estimated to be half of the speed of light ${ }^{6}$. Instead we use a semirelativistic approach based on the the spinless Salpeter type equation

$$
\left[\sqrt{\vec{p}_{q}^{2}+m_{q}^{2}}+\sqrt{\vec{p}_{\bar{q}}^{2}+m_{\bar{q}}^{2}}+V(r)-M\right] \psi(\vec{r})=0
$$

that retains the relativistic kinematics and is suitable for describing the spin-average meson spectrum ( $M=$ mass of the meson) in terms of our static radial potential $V(r)$. Let us note that spin-spin corrections are expected to be relevant for the lowest $s$-wave states. As these corrections are three times bigger for spin singlets than for spin triplets we shall use spin triplet data for approximate comparison. For the low lying $p$-wave and $d$-wave states the absence of spin-orbit and tensor interactions in our potential is expected to deteriorate the fit. For higher states however we expect spin-dependent effects not to be relevant and our approximation to be accurate. 


\begin{tabular}{|cccc|}
\hline$n_{r} L$ & $\left\langle r^{2}\right\rangle^{1 / 2}$ & $M$ & $M_{P D G}$ \\
\hline & $\mathrm{fm}$ & $\mathrm{MeV}$ & $\mathrm{MeV}$ \\
\hline $1 s$ & 0.3 & 3094 & $3096.916 \pm 0.011$ \\
$2 s$ & 0.8 & 3699 & $3686.09 \pm 0.04$ \\
$1 d$ & 0.9 & 3884 & $3772.92 \pm 0.35$ \\
$3 s$ & 1.2 & 4040 & $4039 \pm 1$ \\
$2 d$ & 1.4 & 4154 & $4153 \pm 3$ \\
$4 s$ & 1.7 & 4263 & $4263_{-9}^{+8}$ \\
$3 d$ & 1.9 & 4341 & $4361 \pm 18^{B e}$ \\
& & & $4324 \pm 24^{B a}$ \\
$5 s$ & 2.3 & 4421 & $4421 \pm 4$ \\
$4 d$ & 2.5 & 4477 & \\
$6 s$ & 2.9 & 4537 & \\
$5 d$ & 3.1 & 4578 & \\
$7 s$ & 3.6 & 4625 & \\
$6 d$ & 3.8 & 4656 & \\
& & & $4664 \pm 16^{B a}$ \\
$8 s$ & 4.3 & 4693 & \\
\hline
\end{tabular}

Concerning the parameters, $\sigma$, the string tension, and $\nu$, the string breaking scale both determining the confinement, are taken as usual to be flavour and $\vec{q}^{2}$ independent. $\sigma=0.183 \mathrm{GeV}^{2}$ is fixed from the Regge trajectory for $\rho_{J}$ and $\mathrm{a}_{J}$ and $\nu=2.3 \mathrm{fm}$ from the fit to the static light-quark isovector meson spectrum ${ }^{8}$. The other two parameters, $k_{c}$ and $m_{c}$, are fixed to get a good overall description of charmomium. The calculated spectrum ( $M$ stands for the mass of the states) and root mean square radii, $\left\langle r^{2}\right\rangle^{1 / 2}$, for $k_{c}=132.3 \mathrm{MeV}$.fm and $m_{c}=1474 \mathrm{MeV}$ for $1^{--}$states is shown in Table I as compared to data from ${ }^{1}, M_{P D G}$, unless otherwise stated by means of a superindex: $B e$ for Belle data ${ }^{9}, B a$ for BaBar data ${ }^{10}$.

We see that apart from the $1 d$ state whose description is deficient due to the absence of spin-dependent corrections (our results suggest that these are relevant for $\left\langle r_{c \bar{c}}^{2}\right\rangle_{S R}^{1 / 2} \lesssim 1.1 \mathrm{fm}$ ) the whole spectrum is very well reproduced with an unambiguous assignment of quantum numbers to experimental states. Regarding the experimental candidate at $4664 \mathrm{MeV}$ its identification is more uncertain (it could even correspond to a dynamically generated resonance ${ }^{11}$ what would explain its observation as an isolated state). The precise assignment we get makes us trust our predictions for resonances not yet discovered. Thus we predict $M_{\psi(6 s)} \sim 4537 \mathrm{MeV}$. The discovery of a $\psi(6 s)$ state with such a mass would be of great support to our model (other models, quenched or differently unquenched, predict a much higher mass for this state) although its experimental extraction may be difficult due to its overlapping with neighbor states. This difficulty will increase when approaching the limiting $\operatorname{mass}\left(M_{\text {Limit }}\right)_{c \bar{c}} \simeq m_{c}+m_{\bar{c}}+\sigma \nu=5077 \mathrm{MeV}$. 
Our SRSA has been also applied to other static meson sectors providing a precise description of the known light-quark meson static spectrum excepting $s$-waves as well as of bottomonium excepting the low lying $p$ and $d$-waves. Definite predictions for highly excited states are also derived in these sectors. The accurate description of data in all cases suggest our model might be considered as a simplistic QCD-like hadronic description providing a valid alternative for the calculation of the spectral masses in its domain of applicability (the static region up to the limiting mass).

As a consequence of the peculiar long-distance behaviour of the potential the predicted large-sized meson spectra differ greatly from the one predicted by quenched or differently unquenched quark models. The comparison between the different predictions and data may serve to discriminate among models. To this end we have provided a set of definite predictions for states that either may be discovered in a near future or require dedicated analyses to be extracted from current data. We think an experimental effort along these lines could be well rewarded.

This work has been partially funded by the Spanish MCyT and UE FEDER under Contract No. FPA2007-65748, by the spanish CPAN (CSD2007-00042) and by the Prometeo Program (2009/129) of the Generalitat Valenciana. It is also partly funded by HadronPhisics2, a FP7-IAIP of the EU under Grant 227431.

\section{References}

1. K. Nakamura et al., Review of Particle Physics, J. Phys. G 37, 075021 (2010).

2. N. Isgur, Beyond the adiabatic approximation: The impact of thresholds on the hadronic spectrum, Phys. Rev. D 60, 054013 (1999) and references therein.

3. J. Papavassiliou and J. M. Cornwall, Coupled fermion gap and vertex equations for chiral-symmetry breakdown in QCD, Phys. Rev. D 44, 1285 (1991).

4. V. Mathieu, N. Kochelev and V. Vento, The Physics of Glueballs, Int. J. Mod. Phys. E 18, 1 (2009).

5. D.I. Diakonov and V.Yu. Petrov, A theory of light quarks in the instanton vacuum, Nucl. Phys. B 272 , 457 (1986); P. O. Bowman et al., Infrared and ultraviolet properties of the Landau gauge quark propagator, Nucl. Phys. Proc. Suppl. 128, 23 (2004).

6. E. Eichten, S. Godfrey, H. Mahlke and J. Rosner, Quarkonia and their transitions, Rev. Mod. Phys. 80, 1161 (2008) and references therein.

7. S. S. Afonin, Properties of possible new unflavored mesons below $2.4 \mathrm{GeV}$, Phys. Rev. C 76, 015202 (2007).

8. P. González, Long-distance behavior of the quark-antiquark static potential. Application to light-quark mesons and heavy quarkonia, Phys. Rev. D 80, 054010 (2009); P. González and V. Mathieu, Is the meson spectrum limited?, to appear in Int. J. Mod. Phys. A.

9. X. L. Wang et al., Observation of Two Resonant Structures in $e^{+} e^{-} \rightarrow \pi^{+} \pi^{-} \psi(2 S)$ via Initial-State Radiation at Belle, Phys. Rev. Lett. 99, 142002 (2007).

10. B. Aubert et al., Evidence of a Broad Structure at an Invariant Mass of $4.32 \mathrm{GeV} / \mathrm{c} 2$ in the Reaction $e^{+} e^{-} \rightarrow \pi^{+} \pi^{-} \psi(2 S)$ Measured at BABAR, Phys. Rev. Lett. 98, 212001 (2007).

11. F-K Guo, C. Hanhart and U. Meissner, Evidence that the Y(4660) is an $f_{0}(980) \psi^{\prime}$ bound state, Phys. Lett. B 665, 26 (2008). 